\title{
Design of Multi-threshold Acceleration Switch Based on Matlab
}

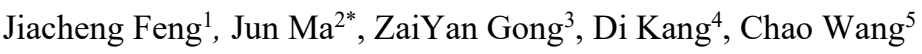 \\ ${ }^{1}$ The Evaluation and Transformation of Sci \& Tech Dept, Chinese Institute of Electronics, No.13 Yuyuantan South Road, Haidian \\ District, Beijing, China \\ ${ }^{2}$ College of Design and Innovation, Tongji University, 1239, Siping Road, Shanghai, China \\ ${ }^{3}$ School of Automotive Studies, Tongji University, 4800, Caoan Road, Shanghai, China \\ ${ }^{4}$ Information Service Department, China Unicom,No.19 Keji 6nd Road,Yanta District,Xi'an, Shaanxi Province, China \\ ${ }^{5}$ Land development and Consolidation Center, Yanchang County Bureau of Land and Resources, No. 7 Xichengyixiang,Yanchang \\ County, Yanan, Shaanxi Province, China
}

\begin{abstract}
Acceleration switch can trigger switches according to the variation of external acceleration without complex control system and circuit. With the excellent characteristics of integration of sensor and actuator, zero power consumption and high reliability, multi-threshold acceleration switch has great application value in the fields of vehicle safety, aerospace and industrial equipment. In order to reduce the complexity of the measurement system and improve the reliability, we realized the design of a multithreshold and anti-overloading acceleration switch which can distinguish specific pulse width. Additionally, the magnetic elastic contact is designed to prevent the rebound phenomenon when switch is overloaded. Considering the coupling force of spring, magnetic elastic contact and magnetic, we built the dynamic model and analysed the influence of the acceleration threshold and the pulse width on the switch actuation. The numerical simulation shows that the proposed switch has accurate sensitivity of acceleration excitation with specific threshold and certain pulse width, and it also has the ability of anti-overload. Consequently, the proposed method provides a new method for designing acceleration switch.
\end{abstract}

\section{Introduction}

In the detection of mechanical equipment, acceleration monitoring is significant, especially in automotive safety, aerospace, industrial robots, engineering vibration measurement and military fields. Accelerometer is a kind of inertial sensor for acceleration measure, and acceleration switch is a kind of inertial device with the function of acceleration sensors and actuators. It has the function of acceleration threshold induction, and the switch can trigger after reaching the design threshold. This paper mainly introduces the design of a multithreshold and anti-overload acceleration switch based on Matlab, and carries out analysis of statics and dynamics.

In recent years, many universities and research institutes have carried out research on acceleration switches, which structure and working principles are varied. For example, B J Hansen ${ }^{[1]}$ designed a bistable micro enclosed accelerometer without power based on the $\mathrm{V}$ shaped beam structure. Under the acceleration excitation, the bistable flexible mechanism can snap to different states. L.J.Currano et al. ${ }^{[2]}$ designed a threeaxis acceleration switch which can be perceived in three directions based on the helical spring structure. A kind of acceleration switch with dual directional adjustable threshold function designed by Kim $\mathrm{H}$ et al. ${ }^{[3]}$ Which can detect bilateral acceleration by a flexible beam structure. At present, the design of acceleration switch mostly uses simple beam structure and single physical field structure, which is difficult to realize multi-stable function, and the contact force is unstable. ${ }^{[4-5]}$

Aiming at the problems of single threshold, unstable contact and slow response of the acceleration switch, a design model of mechanical self-hold and multithreshold bidirectional acceleration switch based on five steady-state mechanism is proposed. An elastic contact with a conductive magnetic film is designed to improve the overload performance. The static and dynamic characteristics of the acceleration switch are analysed based on the magnetic charge model and the elastic mechanics. It is found that the acceleration switch has a characteristic of bidirectional four threshold in statics, and also the response speed is fast. The paper provides a new idea and method for the design of multi-threshold and anti-overload acceleration switch.

\section{Structure and principle}

Figure 1 is a schematic diagram of the multi-threshold acceleration switch structure, which mainly includes mass, extension spring and guide rail structure, pressure spring and guide rail structure, moving magnetic block, fixed magnetic block, low threshold elastic contact, high threshold elastic contact and base. The 3D-effect of the structure is shown in Figure 2. From the diagram, we can see that four magnetic blocks are arranged in a straight 
line. The moving magnetic blocks are inlaid in the middle of mass. The mass with a triangle through-hole can move straight along the guide rail. The mass is connected with an extension spring at both ends of the guide direction. The force between magnetic blocks makes the movement of magnetic blocks shows nonlinear characteristic of force to realize the multistable function. The switch doesn't need external energy when it is in different steady-state. In order to realize switch closure function and anti-overload performance, a kind of elastic contact structure is designed and the magnetic film is set as contact. The mass can be absorbed with the contacts during movement and keep closed in overload environmental.

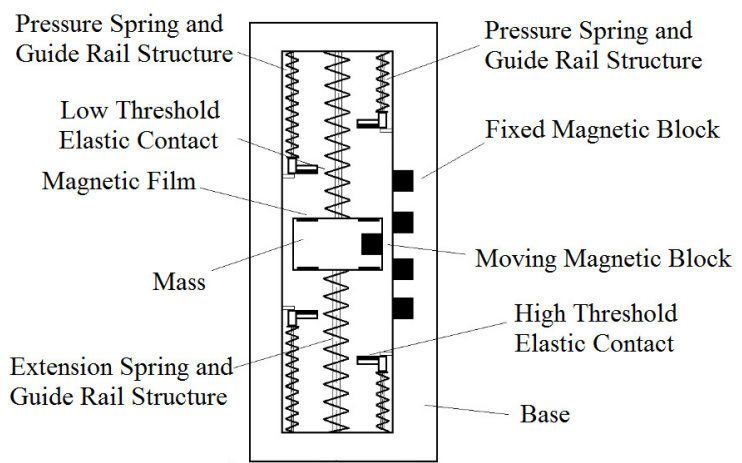

Figure 1. Schematic diagram

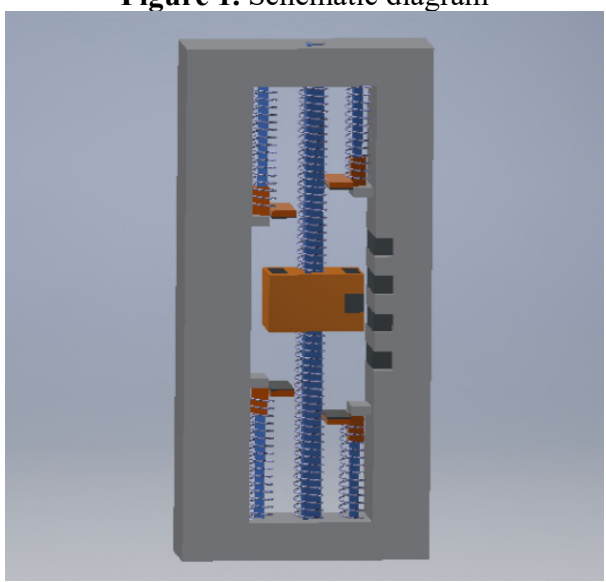

Figure 2. 3D-effect drawing

The mass moves when the external acceleration is loaded. When the acceleration excitation is large enough, the mass snaps to other steady-state and can absorb with the elastic contact to realize switch closure. With different acceleration excitation, the mass can absorb by different contacts to realize the hierarchical closing function. By analyzing the mechanical characteristics of the acceleration switch, the statics and dynamics model are established. According to the design requirements, the material and structural parameters are determined to realize the preset function.

\section{Design and analysis}

\subsection{Statics design and analysis}

In the statics analysis, we takes the moving magnetic block as the research object. Due to the difference situation between the moving magnetic block and elastic contacts, the analysis is divided into three situations. Set $F_{t}$ as the resultant force of mass in the moving direction, $F_{k 1}$ as tension spring resultant force, $F_{k 2}$ as elastic contact force of low threshold, $F_{k 3}$ as elastic contact force of high threshold, $F_{m 1}$ as the resultant force between moving magnetic block and four fixed magnetic blocks, $F_{m 2}$ as the force between magnetic film of mass and magnetic film of low threshold elastic contact, $F_{m 3}$ as the force between magnetic film of mass and magnetic film of high threshold elastic contact.

When both low and high threshold elastic contacts are not absorbed, the physical model is as follows.

$$
F_{t}=F_{k 1}+F_{m 1}+F_{m 2}
$$

When the low threshold elastic contact is absorbed and the high threshold elastic contact is not absorbed, the physical model is as follows.

$$
F_{t}=F_{k 1}+F_{k 2}+F_{m 1}+F_{m 3}
$$

When both low and high threshold elastic contacts are absorbed, the physical model is as follows.

$$
F_{t}=F_{k 1}+F_{k 2}+F_{k 3}+F_{m 1}
$$

In the static analysis, in order to meet the design requirements, the parameters of magnetic film, contact beam and the distance between magnetic blocks are adjusted on the basis of the design threshold. The $a$ is defined as the acceleration excitation, and the $a_{\mathrm{t}}$ is the design threshold, the minimum stable contact force is $P$, the minimum stable contact time is $T$, and the design of the multi-threshold acceleration switch satisfies the following conditions:

(1) when $a<a$ t, the switch is OFF.

(2) when $a \geq a_{\mathrm{t}}$, the switch is OPEN and the response time of the switch is less than $T_{1}$.

(3) when the switch is OPEN, the contact force of the contact is greater than or equal to $P$, and the contact time of the switch is greater than or equal to $T_{2}$.

The resultant force diagram of mass in different directions can be obtained by numerical calculation, as shown in Figure 3. There are 7 zero points of resultant force in the figure which meets the design requirements of multistable function. There are 5 steady-state positions, and the acceleration switch has bidirectional and four threshold characteristics. 


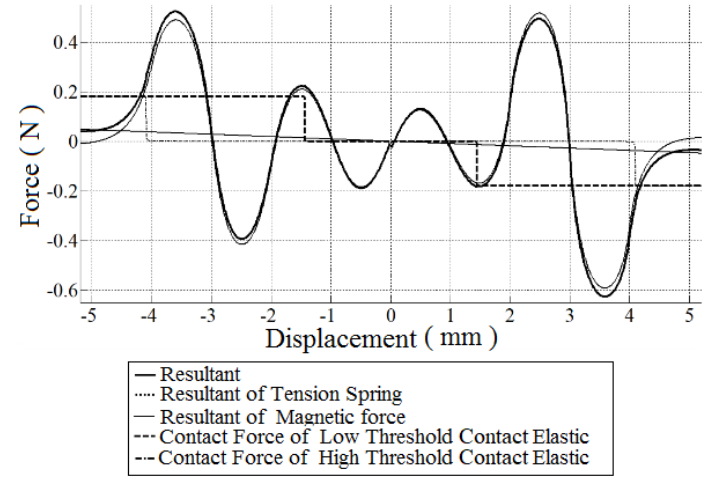

Figure 3. Static force curve of the structure

The static threshold of the acceleration switch is shown as shown in Table 1.

Table 1. Static threshold of acceleration switch

\begin{tabular}{|c|c|c|c|}
\hline \multicolumn{2}{|c|}{ Mass upward movement } & \multicolumn{2}{c|}{ Mass downward movement } \\
\hline Threshold & Data & Threshold & Data \\
\hline$a_{c 1}$ & $3.44 \mathrm{~g}$ & $a_{c 4}$ & $5.01 \mathrm{~g}$ \\
\hline$a_{c 2}$ & $13.27 \mathrm{~g}$ & $a_{c 5}$ & $10.57 \mathrm{~g}$ \\
\hline
\end{tabular}

\subsection{Dynamic design and analysis}

Acceleration switches are working in dynamic environments, so dynamic analysis is significant. The mass is coupled by the coupling effect of inertial force, elastic force, magnetic force, damping force and external excitation $F(\mathrm{t})$ during the motion process. The dynamic model is established according to different working contitions, as shown in the following ${ }^{[6]}$.

When both low and high threshold elastic contacts are not absorbed, the physical model is as follows.

$$
m \ddot{y}+c \dot{y}+k_{1} y=F(t)+F_{m 1}+F_{m 2}
$$

When the low threshold elastic contact is absorbed and the high threshold elastic contact is not absorbed, the physical model is as follows.

$$
m \ddot{y}+c \dot{y}+k_{1} y+k_{2} y=F(t)+F_{m 1}+F_{m 3}
$$

When both low and high threshold elastic contacts are absorbed, the physical model is as follows.

$$
m \ddot{y}+c \dot{y}+k_{1} y+k_{2} y+k_{3} y=F(t)+F_{m 1}
$$

Among them, $y$ is mass movement displacement, $m$ is the mass of mass block, $C$ is damping coefficient, $K_{1}$ is the stiffness coefficient of extension spring, $K_{2}$ is the pressure spring stiffness coefficient of low threshold elastic contact, $K_{3}$ is the pressure spring stiffness coefficient of high threshold elastic contact.

Based on the dynamic model, observing and analyzing the dynamic response of mass under the excitation of rectangular acceleration pulse with different amplitude and the same pulse width. Take the upward acceleration as an example, loading acceleration excitation for mass as shown in Figure 4, the mass block moves upwards. When the external acceleration is equal to the predetermined threshold value $a_{\mathrm{d}}$ $\left(a_{1}=a_{\mathrm{d} 1}, a_{2}=a_{\mathrm{d} 2}\right)$, as shown in the solid line in Figure 4, the mass snaps upwards to the adjacent steady-state, and the switch is closed.

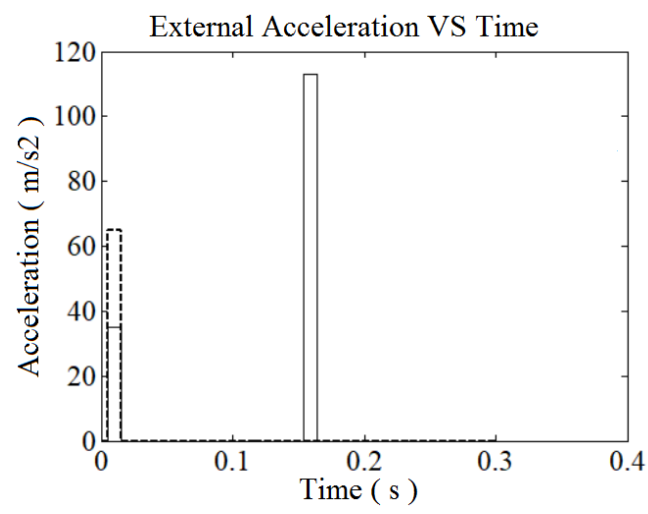

Figure 4. Input acceleration excitation

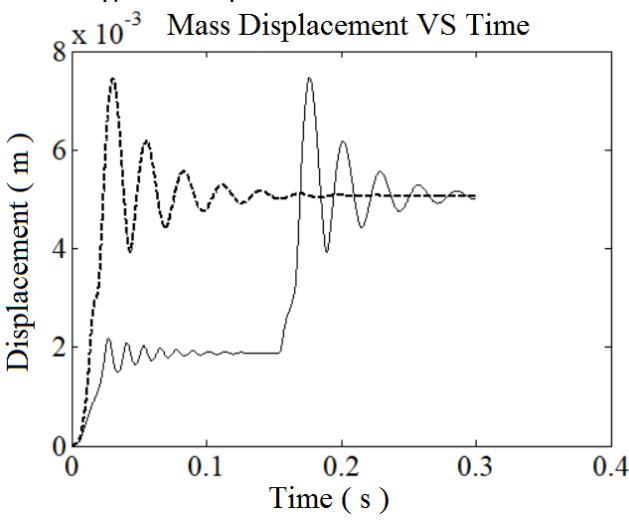

Figure 5. Displacement of the mass

When the external excitation acceleration is $4.8 \mathrm{~g}$ smaller than the threshold acceleration $a_{d 2}$, as shown in the dotted line in Figure 4, the mass snaps from the first steady-state to the third steady-state directly, and the third acceleration threshold is found, we defined it as $a_{\mathrm{d} 3}$.

Fig. 6 and Fig. 7 are the contact displacement curves after loading the acceleration excitation, which reflect the dynamic response of the elastic contacts. As shown in Fig. 6, when the external acceleration is equal to the design threshold, the low threshold elastic contact and the mass block quickly absorb and move together. As shown in Fig. 7, the contact force of the low threshold elastic contact is large enough and stable. We define the time from acceleration loading to contact closure as response time. When the external excitation pulse width is $10 \mathrm{~ms}$, the response time of the system is $18 \mathrm{~ms}$, which meets the design requirements.

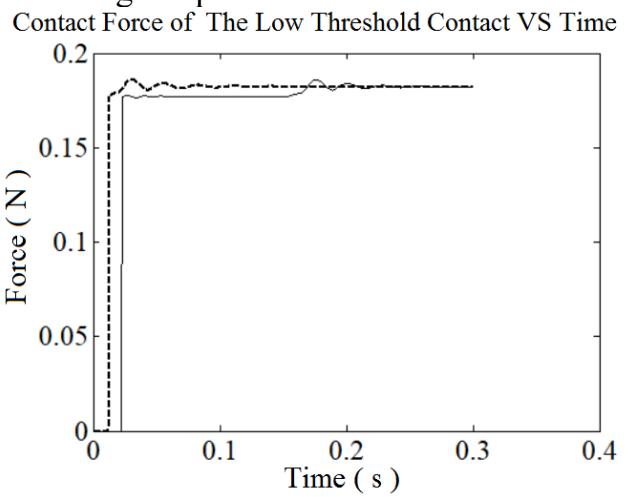

Figure 6. Contact force of the low threshold contact 


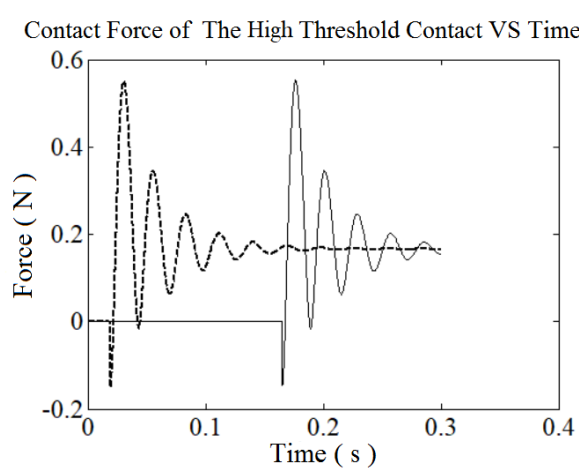

Figure 7. Contact force of the high threshold contact

The threshold values of the acceleration switch under the rectangular acceleration pulse of same pulse width are shown in Table 3.

Table 2. Dynamic threshold of acceleration switch

\begin{tabular}{|c|c|c|c|}
\hline \multicolumn{2}{|c|}{ Mass upward movement } & \multicolumn{2}{c|}{ Mass downward movement } \\
\hline Threshold & Data & Threshold & Data \\
\hline$a_{d 1}$ & $3.57 \mathrm{~g}$ & $a_{d 4}$ & $4.69 \mathrm{~g}$ \\
\hline$a_{d 2}$ & $11.53 \mathrm{~g}$ & $a_{d 5}$ & $9.29 \mathrm{~g}$ \\
\hline$a_{d 3}$ & $6.63 \mathrm{~g}$ & $a_{d 6}$ & $6.93 \mathrm{~g}$ \\
\hline
\end{tabular}

In order to study the overload working effect, we set the excitation amplitude to $a=2 a_{\mathrm{d} 3}$. Simulation analysis result shows that the contact force is large enough and stable, the design requirements are met under the overload environment.

\section{CONCLUSION}

(1) Through the study of the linear layout of the permanent magnet and the nonlinear characteristics of the permanent magnet, and combined with the common analysis of the function of acceleration switch, an antioverload and multi-threshold acceleration switch is designed.

(2) Considering the influence of magnetic force, elastic force and structural damping on the calculation model, a static analysis model of acceleration switch is established, and the static force curve of the structure is obtained by numerical simulation.

(3) The dynamic analysis model of the acceleration switch is established, and the response of the system under the specific acceleration excitation is simulated. It is proved by dynamic analysis that the acceleration switch meets the design requirements.

(4) The overload environment of the acceleration switch is analysed to ensure engineering reliability.

\section{References}

1. Hansen B J, Carron C J, Jensen B D, et al. Plastic latching accelerometer based on bistable compliant mechanisms[J]. Smart Materials and Structures, 2007, 16(5): 1967.

2. 2.L. J. Currano, C. R. Becker, G. L. Smith, B. Isaacson and C. J. Morris, "3-Axis acceleration switch for traumatic brain injury early warning," Micro Electro Mechanical Systems (MEMS), 2012
IEEE 25th International Conference on, Paris, 2012, pp. 484-487.

3. Kim H, Jang $\mathrm{Y} H$, Kim $\mathrm{Y} \mathrm{K}$, et al. MEMS acceleration switch with bi-directionally tunable threshold[J]. Sensors and Actuators A: Physical, 2014, 208: 120-129..

4. 4.J. Zhao, J. Jia and G. Chen, "A Novel MEMS Parallel-beam Acceleration Switch," 2006 2nd IEEE/ASME International Conference on Mechatronics and Embedded Systems and Applications, Beijing, 2006, pp. 1-5.

5. Zhao J, Liu P, Tang Z, et al. A Wireless MEMS Inertial Switch for Measuring Both Threshold Triggering Acceleration and Response Time[J]. Instrumentation and Measurement, IEEE Transactions on, 2014, 63(12): 3152-3161.

6. Howell L L. Compliant Mechanisms[M]// 21st Century Kinematics. Springer London, 2013:457463. 